\title{
Utility of CT Perfusion Imaging for Grading of Clear Cell Renal Cell Carcinoma
}

Yue-Lang Zhang ${ }^{1}$, Juan Ren ${ }^{2 *}$, Bo-Lang Yu ${ }^{1 *}$, Kai Qu${ }^{1}$, Ke Wang ${ }^{1}$, Yong-Qian Qiang ${ }^{1}$, Chen-Xia Li ${ }^{1}$, Xing-Wang Sun ${ }^{1}$ and Zongfang Li ${ }^{3}$

${ }^{1}$ Department of Imaging, First Affiliated Hospital, Medicine School of Xi'an Jiaotong University, Xi'an 710061, Shaanxi, PR China

${ }^{2}$ Department of Radiotherapy, Cancer center, First Affiliated Hospital, Medicine School of Xi'an Jiaotong University, Xi'an 710061, Shaanxi, PR China

${ }^{3}$ Department of General Surgery, Second Affiliated Hospital, Medicine School of Xi'an Jiaotong University, Xi'an 710004, Shaanxi, PR China

\begin{abstract}
Objective: To investigate the correlation between 64-slice spiral CT perfusion imaging of clear cell renal cell carcinoma (CCRCC) and Fuhrman's nucleus grading.

Methods: A total of 54 cases of pathologically confirmed CCRCC were consecutively included, and underwent 64-slice spiral CT perfusion imaging. The perfusion fraction (PF) of the contralateral and ipsilateral part of kidney and the tumor was measured respectively. The peak enhancement intensity (PEI) and blood volume (BV) were measured as well. T-test was performed in PF, BV and PEI value between contralateral kidney and CCRCC, between low-grade and high-grade CCRCC. Receiver operating characteristic (ROC) curves were used to evaluate the diagnostic performance of PF and BV in distinguishing clear cell carcinoma with different grade. The diagnostic accuracy of PF and BV was also tested after conventional CT screening.
\end{abstract}

Results: There were significant differences in PF, PEI and BV between the contralateral kidney and renal cell carcinoma $(p<0.05)$. There were also significant differences in PF and BV between low-grade and high-grade clear cell carcinoma $(p<0.05)$. No significant difference was found in PEI between low-grade and high-grade renal cell carcinoma ( $p>0.05)$. Single or combined use of the PF and BV showed low accuracy in grading of clear cell carcinoma. The diagnostic accuracy of PF and BV in clear cell carcinoma will be improved by the combination of the perfusion $\mathrm{CT}$ and routine CT application.

Conclusion: PF and BV values are significantly higher in high-grade CCRCC than in low-grade CCRCC, and their application will increase the accuracy for detecting high-grade CCRCC than conventional CT scan alone.

Keywords: Computed tomography (CT); Computed tomography perfusion; low-grade CCRCC; high-grade CCRCC

\section{Introduction}

Clear cell renal cell carcinoma (CCRCC) accounts for about 63 $\sim 88.7 \%$ of the renal cell carcinoma (RCC) [1,2]. In addition to traditional surgery, minimally invasive treatments, including radio frequency ablation and cryoablation surgery, are also choices for some small, low-grade clear cell carcinoma. These alternative therapies are especially suitable for the elderly, whose postoperative quality of life can be significantly improved $[3,4]$. Therefore, accurate evaluation of tumor grading is essential for the treatment plan, the choice of surgical approach and objective evaluation of prognosis. Currently, in CCRCC, Fuhrman nuclear grading system is superior to tumor size, stage and other prognostic factors, and is considered to be a quite accurate prognostic evaluation system [5-7].

Clear cell carcinoma grading must be pathologically confirmed after surgery, which makes it little preoperative guiding significance. Computed tomography (CT) and magnetic resonance imaging (MRI) are currently the main imaging methods, but are of limited value in the grading evaluation of renal cell carcinoma [8,9]. Jain R et al. [10], showed that PS and CBV showed strong association with gliomas grading, and high-grade gliomas have higher perfusion parameters such as PS and CBV compared with low-grade gliomas. Ding B et al. [11], suggested that perfusion $\mathrm{CT}$ is able to contribute to the grading of gliomas. Xiong $\mathrm{Z}$ et al. [12], thought that perfusion characteristics of CT are helpful to determine lung cancer differentiation. Sahani DV et al. [13], suggested that the CT perfusion of well differentiated hepatocellular carcinoma is higher than that of moderately differentiated and poorly differentiated hepatocellular carcinoma. However, CT perfusion has not been reported in the staging of CCRCC.

Therefore, this study used 64-slice spiral CT perfusion imaging to explore perfusion characteristics of CCRCC, and compared tumor classification with Fuhrman's nucleus grading system. Our study aims to explore their correlation from the micro-cycle perspective, and to provide help for CCRCC grading by functional imaging.

\section{Materials and Methods}

\section{Patients}

The research program has been discussed, approved and recorded by Medicine Ethics Committee of the First Affiliated Hospital of Xi'an Jiaotong University. All subjects have signed the informed consent form of Enhanced CT. The hospitalized patients with suspicion of renal tumor were forward-looking consecutively enrolled from March 1, 2010 to March 30, 2011. The inclusion criteria for patients were: (1) renal tumors identified by B-ultrasonography, CT or MRI scanning; (2) Good renal function and cooperation with the inspection. Exclusion criteria were patients with heart or kidney dysfunction; unresectable tumor due to metastasis of other organs.

${ }^{*}$ Corresponding authors: Bo-Lang Yu, PhD, Department of Imaging, Firs Affiliated Hospital, Medicine School of Xi'an Jiaotong University, Xi'an 710061, Shaanxi, PR China, Tel: +086-029-85225009; Fax: +086-029-85225009; E-mail: cjr.yubolang@vip.163.com

Juan Ren, Department of Radiotherapy, Cancer center, First Affiliated Hospital, Medicine School of Xi'an Jiaotong University, Xi'an 710061, Shaanxi, PR China, Tel: +086-029-85323249; E-mail renjuan88@yahoo.com.cn

Received February 29, 2012; Accepted March 31, 2012; Published April 04, 2012

Citation: Zhang YL, Ren J, Yu BL, Qu K, Wang K, et al. (2012) Utility of CT Perfusion Imaging for Grading of Clear Cell Renal Cell Carcinoma. Hereditary Genetics 1:105. doi:10.4172/2161-1041.1000105

Copyright: @ 2012 Zhang YL, et al. This is an open-access article distributed under the terms of the Creative Commons Attribution License, which permits unrestricted use, distribution, and reproduction in any medium, provided the original author and source are credited. 


\section{Inspection methods}

All subjects were inspected by 64-slice CT scanning (Philips Brilliance). Respiratory training was performed before the subjects received inspection. The patients were positioned supine with pressurized cummerbund, and performed routine kidney scanning, then the maximum level of lesion was taken as center for scanning with the upper and lower range $4 \mathrm{~cm}$. With a $21 \mathrm{G}$ catheter placed in antecubital vein, a bolus of $50 \mathrm{ml}$ non-ionic contrast agent was injected through high-pressure syringe (IohexolInjection $300 \mathrm{mgI} / \mathrm{ml}$, Ulrich, Germany), with injection rate of $5.0 \mathrm{ml} / \mathrm{s}$. Continuous dynamic multilayer film scan mode was performed, and the scan conditions were 110 $\mathrm{kV}$ for the tube voltage and $80 \mathrm{~mA}$ for tube current, then automatically scanning was trigger $5 \mathrm{~s}$ after injection. Scanning was performed in the quiet breathing condition at intervals of $1.5 \mathrm{~s}$ with thickness of $2.5 \mathrm{~mm}$, and cycle of scanning was 30 times, and a total of 480 images were obtained. Again after the $90 \mathrm{~s}$ and $180 \mathrm{~s}$, renal scanning was once again performed to obtain images of renal medulla and delayed phase, respectively.

\section{post-processing of perfusion imaging parameters}

The perfusion raw data were input software workstation (Philips Extended Brilliance Workspace, V4.5.2) for processing. The same level abdominal aorta artery was selected as a reference artery, and regions of interest were selected in the abdominal aorta center to acquire the enhancement of the abdominal aorta, the time to peak (TTP) value, and time-density curves (TDC) value. Threshold parameters were set as following: minimum CT value was $-100 \mathrm{HU}$, maximum CT value was $300 \mathrm{HU}$; pseudo-color perfusion parametric maps of each pixel were ultimately obtained within the threshold range, including perfusion fraction (PF), blood volume (BV), and peak enhancement intensity (PEI).

\section{Data measurement}

Blinded data measurements were performed by two doctors of imaging department (a MD, deputy director, with 14 years work

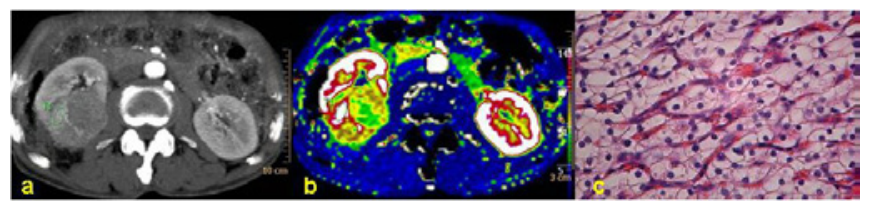

Figure 1: Clear cell carcinoma of right kidney, grade II. (a) tMIP image: round strengthening shadow in dorsal side of right kidney, with clear boundary, and low density was shown in the eccentric necrotic area. (b) Lesions on perfusion map showed white high perfusion shadow, ROIT1: $P$ : $143.33 \mathrm{ml} / \mathrm{min} / 100 \mathrm{~g}$ PEI: $243.35 \mathrm{Hu}, \mathrm{BV} 42.72 \mathrm{ml} / 100 \mathrm{~g}$. (c) Pathological picture, HE $\times 400$. Clear Cytoplasm was seen in tumor cells. The size of nucleus was similar. Nucleolus was seldom seen.

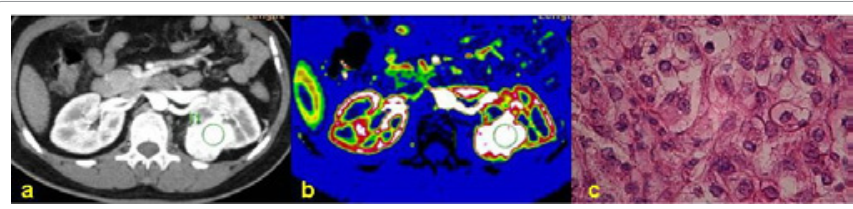

Figure 2: Clear cell carcinoma in right kidney, grade III. (a) tMIP image: significantly strengthen irregular film in dorsal side of left kidney. (b) perfusion of lesions showed white high perfusion shadow, ROIT1: P: $193.72 \mathrm{ml} / \mathrm{min} / 100 \mathrm{~g}$, PEl: $340.54 \mathrm{Hu}, \mathrm{BV}: 80.42 \mathrm{ml} / 100 \mathrm{~g}$. Pathological picture: HE $\times 400$. Clear Cytoplasm was seen in tumor cells. The size of nucleus was uneven. Nucleolus was frequently seen. experience; a physician, with six years of work experience, engaged in the study of kidney MRI functional imaging in a graduate student). In perfusion map, the window width and window level were adjusted with tMIP as basis and the contralateral kidney as control. The maximum level of the tumor was selected and ROI was manually delineated including the most obvious substantial part of tumor enhancement. Regions of tumor necrosis, liquefaction or fat content were excluded, and high perfusion area was chosed as far as possible, referring to the color of the perfusion pseudo-color pictures (Figures 1a-c,2a-c). The contralateral renal cortex was manually delineated and perfusion parameters of contralateral renal cortex and renal space-occupying lesions were obtained, including PF, BV and PEI, and their mean values were averaged from two measurements.

\section{Histological analysis}

All postoperative pathological slices were graded by a deputy director of Department of Pathology with 15 years of experience, in accordance with the classification criteria of the Fuhrman grading system (I-IV). Grades I and II were considered low-grade clear cell carcinoma, grades III and IV were considered high-grade clear cell carcinoma $[14,15]$.

\section{Statistical analysis}

The experimental results are presented as mean \pm standard deviation. T-test was applied in PF, BV and PEI values between the contralateral renal cortex and medulla, and clear cell carcinoma. T-test was also applied in PF, BV and PEI values between low-grade and high-grade clear cell carcinoma. Receiver operating characteristic (ROC) curve was applied to evaluate diagnostic efficacy of PF and BV in degrading clear cell carcinoma. Conventional CT plain scanning and enhanced CT scanning were assessed in nuclear grade using the chisquare test. ROC curve was used to evaluate diagnostic accuracy of CT perfusion after conventional CT in Low-grade and high-grade clear cell carcinoma. SPSS 16.0 statistical software (SPSS, Inc., Chicago, IL, USA) was applied, and $\mathrm{P}<0.05$ was considered statistically significant.

\section{Results}

\section{Lesions}

Based on inclusion and exclusion criteria, a total of 60 cases (41 cases of male, 19 cases of female, 33-79 years old, 58 years median age) of hospitalized patients with suspected renal cell carcinoma were performed 64-slice CT perfusion imaging. Renal cell carcinoma was confirmed by pathology within an average of 12 days after inspection (range 2-46 days) (46 patients with radical nephrectomy; 14 patients with nephron-sparing resection), of which 54 cases of clear cell carcinoma were included in this study. Six cases were excluded ( 2 cases were chromophobe cell tumor, 1 case was unclassified stromal tumors, 1 case was metastatic renal adenocarcinoma, 2 cases were papillary renal cell carcinoma). Among all 54 cases of clear cell carcinoma, 34 cases were low-grade ( 5 cases of grade I, 29 cases of grade II) clear cell carcinoma, with tumor size of $1.4 \sim 9.2 \mathrm{~cm}$, and 20 cases were highgrade (17 cases of grade III, 3 cases of grade IV), with tumor size of 3.0 $\sim 10.4 \mathrm{~cm}$. A total of 54 lesions in 54 patients were included in statistical analysis, comprising 34 low-grade and 20 high-grade CCRCC.

\section{Comparisons in PF, PEI, BV values between contralateral kidney and renal cell carcinoma}

PF, PEI, BV values of contralateral kidney cortex were greater than those of the renal medulla. PF, PEI, BV values of contralateral kidney cortex were significantly higher than those of renal cell carcinoma, with 
Citation: Zhang YL, Ren J, Yu BL, Qu K, Wang K, et al. (2012) Utility of CT Perfusion Imaging for Grading of Clear Cell Renal Cell Carcinoma. Hereditary Genetics 1:105. doi:10.4172/2161-1041.1000105

Page 3 of 5

\begin{tabular}{|c|c|c|c|c|}
\hline $\begin{array}{c}\text { CT perfusion } \\
\text { parameters }\end{array}$ & $\begin{array}{c}\text { Contralateral } \\
\text { kidney cortex }\end{array}$ & $\begin{array}{c}\text { Contralateral } \\
\text { kidney medulla }\end{array}$ & $\begin{array}{c}\text { Clear cell } \\
\text { carcinoma }\end{array}$ & $\mathrm{P}$ \\
\hline $\mathrm{PF}(\mathrm{m} / \mathrm{min} / 100 \mathrm{~g})$ & $185.42 \pm 40.92$ & $32.56 \pm 10.46$ & $140.48 \pm 39.06$ & 0.000 \\
\hline $\mathrm{BV}(\mathrm{m} / 100 \mathrm{~g})$ & $44.33 \pm 10.54$ & $23.26 \pm 13.34$ & $36.41 \pm 10.00$ & 0.000 \\
\hline $\mathrm{PEI}(\mathrm{Hu})$ & $205.14 \pm 33.83$ & $54.02 \pm 14.27$ & $154.65 \pm 42.85$ & 0.000 \\
\hline
\end{tabular}

Table1: Comparison in PF, PEI, BV values between contralateral kidney cortex and renal cell carcinoma

\begin{tabular}{|l|c|c|c|}
\hline CT perfusion parameters & Low-grade CCRCC & High-grade CCRCC & $p$ \\
\hline PF $(\mathrm{m} / \mathrm{min} / 100 \mathrm{~g})$ & $127.67 \pm 32.60$ & $159.98 \pm 23.05$ & 0.003 \\
\hline BV $(\mathrm{m} / 100 \mathrm{~g})$ & $32.63 \pm 9.73$ & $40.59 \pm 9.05$ & 0.038 \\
\hline PEI $(\mathrm{Hu})$ & $159.68 \pm 40.63$ & $159.98 \pm 23.06$ & 0.868 \\
\hline
\end{tabular}

Table 2: Comparison in PF, PEI, BV values between Low-grade CCRCC and Highgrade CCRCC.

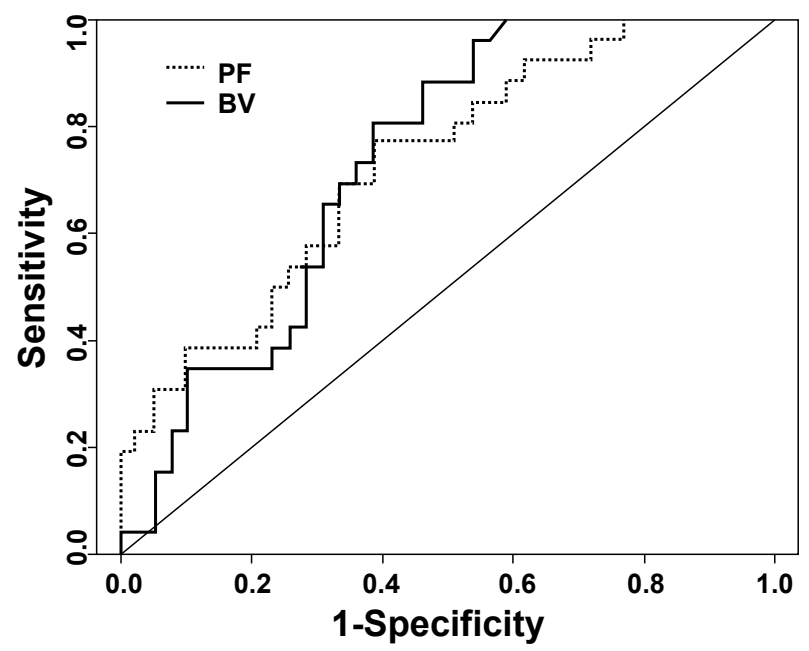

Figure 3: ROC curve of separate evaluation of clear cell carcinoma grading by PF or BV. Areas under the curve were 0.729 in the PF and 0.734 in BV, with no statistically significant difference $(p>0.05)$

significant difference $(\mathrm{p}<0.05)$ (Table 1$)$.

\section{Comparisons in PF, PEI, BV values between different grades of clear cell carcinoma}

$\mathrm{PF}$ and BV values of high-grade clear cell carcinoma were higher than those of low-grade clear cell carcinoma, with statistically significant difference $(\mathrm{p}<0.05)$. No significant difference was found in PEI between the two groups ( $p>0.05$ ) (Table 2).

\section{Evaluation in identification of different grades of clear cell carcinoma using PF and BV measured values}

ROC curve was used to evaluate clear cell carcinoma grading by $\mathrm{PF}$ or BV alone (Figure 3). As independent diagnostic markers, area under the curve of PF and BV was 0.729 and 0.734 , respectively, with no significant difference $(\mathrm{p}>0.05)$.

The gradient effects of clear cell carcinoma was evaluated by alone or combined PF (cut-off point value of 136.88) and BV values (cut-off point value 32.17). The results showed that the parallel combination of $\mathrm{PF}$ and BV has highest sensitivity (96.15\%); series combination of PF and BV has highest specificity (71.79\%). BV values alone showed the highest Youden index (0.42) for diagnosis, and was still below the 0.5 standard, indicating poor clinical utility.

\section{Evaluation of nuclear grading between conventional CT plain scanning and enhanced CT scanning}

The size of the low-grade clear cell carcinoma was $4.66 \pm 2.07 \mathrm{~cm}$, high-grade clear cell carcinoma was $5.35 \pm 2.20 \mathrm{~cm}$, and there was significant difference $(\mathrm{p}<0.05)$ but overlap between the two sets of data. Comparison of conventional CT features between low-grade and high-grade CCRCC was listed in Table 4. Conventional CT features of the tumor showed that in addition to fatty infiltration, there were no statistically differences in shape, necrosis, hemorrhage, calcification, invasion in renal pelvis and renal vein, and enhancement homogeneity between low-grade and high-grade CCRCC identification.

\section{Evaluation of low and high-grade clear cell carcinoma by} joint conventional CT and CT perfusion

We further selected fatty infiltration as a hierarchical standard in conventional CT, and based on this standard, PF and BV measurement values were used to identify different grading in clear cell carcinoma. In group with fatty infiltration, the area under the curve of PF and BV were 0.912 and 0.819 , respectively (Figure $4 \mathrm{a}$ ) while in group without fatty infiltration, the area under the curve of PF and BV values were 0.403 and 0.641 , respectively (Figure $4 \mathrm{~b}$ ). The above data showed that the pre-stratification by whether there is fatty infiltration, can effectively increase the area under the ROC curve of the PF and BV. Based on alone or combined PF (cut-off point value of 139.46) and BV (cutoff point value 33.33), the effects on renal cell carcinoma grading were evaluated in fatty infiltration group. The results showed that Youden index was greater than 0.5 whether single or joint $\mathrm{PF}$ and $\mathrm{BV}$ values were used for diagnosis; Youden index was highest (0.78) when single $\mathrm{PF}$ value was used for diagnosis.

\section{Discussion}

In this study, we found that the PV, PEI and BV values of the normal contralateral kidney were significantly higher than those of clear cell carcinoma. PF and BV value of low-grade clear cell carcinoma were lower than those of high-grade clear cell carcinoma. Combined

\begin{tabular}{|c|c|c|c|}
\hline & Sensitivity (\%) & Specificity (\%) & Youden index \\
\hline BV & 88.46 & 53.85 & 0.42 \\
\hline PF & 76.92 & 61.54 & 0.38 \\
\hline $\begin{array}{c}\text { Parallel combina- } \\
\text { tion }\end{array}$ & 69.23 & 71.79 & 0.41 \\
\hline Series combination & 96.15 & 43.59 & 0.40 \\
\hline
\end{tabular}

Table 3: Evaluation of renal cell carcinoma grading by separate and joint use of $\mathrm{BV}$ and $\mathrm{PF}$ values.

\begin{tabular}{|c|c|c|c|}
\hline Feature & $\begin{array}{l}\text { Low-grade } \\
\operatorname{ccRCC}(\%)\end{array}$ & $\begin{array}{l}\text { High-grade } \\
\operatorname{ccRCC}(\%)\end{array}$ & $P$ \\
\hline Irregular margin & $79.41(27 / 34)$ & $80.00(16 / 20)$ & 0.623 \\
\hline Necrosis & $82.35(28 / 34)$ & $90.00(18 / 20)$ & 0.366 \\
\hline Hemorrhage & $8.82(3 / 34)$ & $5.00(1 / 20)$ & 0.525 \\
\hline Calcification & $5.88(2 / 34)$ & $0(0 / 20)$ & 0.392 \\
\hline $\begin{array}{l}\text { Perinephric fatty in- } \\
\text { vasion }\end{array}$ & $8.82(3 / 34)$ & $40.00(8 / 20)$ & 0.012 \\
\hline $\begin{array}{l}\text { Renal pelvis inva- } \\
\text { sion }\end{array}$ & $11.77(4 / 34)$ & $25.00(5 / 20)$ & 0.188 \\
\hline Renal vein invasion & $8.82(3 / 34)$ & $20.00(4 / 20)$ & 0.221 \\
\hline $\begin{array}{l}\text { Enhancement ho- } \\
\text { mogeneity }\end{array}$ & $73.53(25 / 34)$ & $90(18 / 20)$ & 0.134 \\
\hline
\end{tabular}

Table 4: Comparison of Conventional CT Features for Low-Grade and High-Grade Clear Cell Renal Cell Carcinoma. 

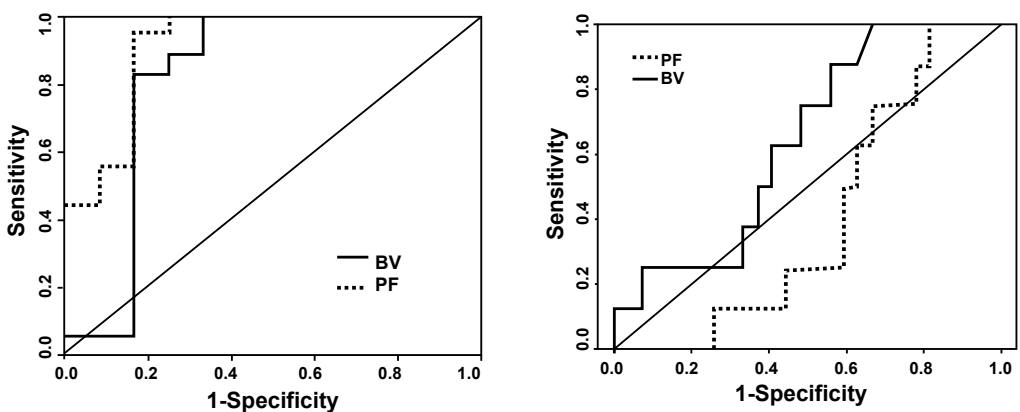

Figure 4: (a) Grading of renal cell carcinoma with fatty infiltration, the area under the curve of PF and BV were 0.912 and 0.819 , respectively; (b) Grading of renal cell carcinoma without fatty infiltration, the area under the curve of PF and BV were 0.403 and 0.641 , respectively.

\begin{tabular}{|c|c|c|c|}
\hline & Sensitivity (\%) & Specificity (\%) & Youden index \\
\hline BV & 88.46 & 53.85 & 0.42 \\
\hline PF & 76.92 & 61.54 & 0.38 \\
\hline Parallel combination & 69.23 & 71.79 & 0.41 \\
\hline Series combination & 96.15 & 43.59 & 0.40 \\
\hline
\end{tabular}

[19]. These factors can reduce BF, PEI and BV values in cancer substantive, making lower perfusion in renal cell carcinoma compared with normal renal cortex.

The PF and BV in low-grade clear cell carcinoma were statistically significant lower than those in high-grade clear cell carcinoma ( $\mathrm{p}$ $<0.05$ ), which is similar to the perfusion in liver cancer grading performance [10]. Brain gliomas are different from tumors of the body organs [20], and there are difference in hemodynamic changes in tumors with different nature or different degree of differentiation. Higher tumor grade means higher malignancy, lower differentiation, more tumor angiogenesis, and less complete tumor vascular endothelial cells. Therefore, these characteristics should indirectly reflected by CT perfusion. No significant difference was found in PEI between lowgrade and high-grade tumor, and shows there is no obvious correlation between degree of enhancement and tumor grading.

Combined use of conventional CT and perfusion imaging may contribute to the diagnosis of CCRCC grading. Compared with renal cell carcinoma grading, tumor size makes significant differences in various features of the conventional CT. Although tumor sizes with different grades are overlapping, small tumors may be high-grade and large tumors may also be low-grade. However, the overall trend is that tumor mean size increases with the increase in tumor grade. This is also consistent with the tumor evolution that tumor will acquire more and more invasive characteristics in tumor growth process. Apart from perirenal fatty infiltration, conventional CT characteristic morphology of the tumor, such as necrosis, cystic degeneration, hemorrhage, calcification, renal pelvis and renal vein violations, and enhanced heterogeneity make no statistical difference in identifying low-grade and high-grade CCRCC. Literature [7], reported that the tumor size, necrosis, and surrounding fat infiltration inspected by conventional MRI will help in diagnosis of high-grade clear cell carcinoma. We took fatty infiltration in conventional CT as a hierarchical standard: in group with fatty infiltration, the area under the curve of PF and BV were 0.912 and 0.819 , respectively; and in group without fatty infiltration, the area under the curve of PF and BV were 0.403 and 0.641 , respectively. This indicates that pre-stratification with existence of fatty infiltration can effectively improve the sensitivity and specificity of the PF and BV in grading of clear cell carcinoma.

Compared with the conventional CT, perfusion imaging can indeed provide more information about lesions, and its value is gradually being revealed. The scanning range of CT perfusion is limited. Therefore, it only reflects hemodynamics of part of the organ, and can not provide an accurate, real-time and full reflection on the organ. 
Citation: Zhang YL, Ren J, Yu BL, Qu K, Wang K, et al. (2012) Utility of CT Perfusion Imaging for Grading of Clear Cell Renal Cell Carcinoma. Hereditary Genetics 1:105. doi:10.4172/2161-1041.1000105

Page 5 of 5

\section{Conclusion}

PF and BV values are significantly higher in high-grade CCRCC than in low-grade CCRCC, and their application will increase the accuracy for detecting high-grade CCRCC than conventional CT dose alone.

\section{Grant Support:}

This manuscript is supported by the National Natural Science Foundation of China (Juan Ren, 30973175 and 81172490), Scientific Research Foundation for Returning Scholars of Education Ministry of China (Juan Ren, 0601-18920006) Scientific and Technological Research Foundation of Shaanxi Province (Juan Ren, 2007K09-09, 2012K13-01-06), Program for Changjiang Scholars and Innovative Research Team in University (Zongfang Li, PCSIRT:1171).

\section{References}

1. Delahunt B, Bethwaite PB, Nacey JN (2007) Outcome prediction for renal cell carcinoma: evaluation of prognostic factors for tumours divided according to histological subtype. Pathology 39: 459-465

2. Patard JJ, Leray E, Rioux-Leclercq N, Cindolo L, Ficarra V, et al. (2005) Prognostic value of histologic subtypes in renal cell carcinoma: a multicenter experience. J clin oncol 23: 2763-2771.

3. Van Poppel H, Becker F, Cadeddu JA, Gill IS, Janetschek G, et al. (2011) Treatment of localised renal cell carcinoma. Eur urol 60: 662-672.

4. Clements T, Lin YK, Raman JD (2011) Current status of ablative techniques for small renal masses. Expert rev anticancer ther 11: 879-891.

5. Ficarra V, Righetti R, Martignoni G, Amico AD, Pilloni S, et al. (2001) Prognosticvalue of renal cell carcinoma nuclear grading:multivariate analysis of 333 cases. Urol Int 67: 130-134.

6. Gudbjartsson T, Hardarson S, Petursdottir V, Thoroddsen A, Magnusson J, et al. (2005) Histological subtyping and nuclear grading of renal cell carcinoma and their implications for survival: a retrospective nation-wide study of 629 patients. Eur Urol 48: 593-600.

7. Minardi D, Lucarini G, Mazzucchelli R, Milanese G, Natali D, et al. (2005) Prognostic role of Fuhrman grade and vascularendothelial growth factor in pT1a clear cell carcinomain partial nephrectomy specimens. J Urol 174: 12081212.

8. Birnbaum BA, Bosniak MA, Krinsky GA, Cheng D, Waisman J, et al. (1994) Renal cell carcinoma: correlation of CT findings with nuclear morphologic grading in 100 tumors. Abdom imaging 19: 262-266.
9. Pedrosa I, Chou MT, Ngo L, H Baroni R, Genega EM, et al. (2008) MR classification of renal masses with pathologic correlation. Eur Radiol 18: 365 375 .

10. Jain R, Ellika SK, Scarpace L, Schultz LR, Rock JP, et al. (2008) Quantitative Estimation of Permeability Surface Area Product in Astroglial Brain Tumors Using Perfusion CT and Correlation with Histopathologic Grade. AJNR Am j neuroradiol 29: 694-700.

11. Ding B, Ling HW, Chen KM, Jiang H, Zhu YB (2006) Comparison of cerebral blood volume and permeability in preoperative grading of intracranial glioma using CT perfusion imaging. Neuroradiology 48: 773-781.

12. Xiong Z, Liu JK, Hu CP, Zhou H, Zhou ML, et al. (2010) Role of Immature Microvessels in Assessing the Relationship between CT Perfusion Characteristics and Differentiation Grade in Lung Cancer. Arch med res 41: 611-617.

13. Sahani DV, Holalkere NS, Mueller PR, Zhu AX (2007) Advanced Hepatocellular Carcinoma: CT Perfusion of Liver and Tumor Tissue-Initial Experience. Radiology 243: 736-743.

14. Lang $\mathrm{H}$, Lindner $\mathrm{V}$, de Fromont $\mathrm{M}$, Molinié $\mathrm{V}$, Letourneux $\mathrm{H}$, et al. (2005) Multicenter determination of optimal interobserver agreement using the Fuhrman grading system for renal cell carcinoma:assessment of 241 patients with > 15-yearfollow-up. Cancer 103: 625-629.

15. Bektas S, Bahadir B, Kandemir NO, Barut F, Gul AE, et al. (2009) Intraobserver and interobserver variability of Fuhrman and modified Fuhrman grading systems for conventional renal cell carcinoma. Kaohsiung J Med Sci 25: 596600 .

16. Chen Y, Zhang J, Dai J, Feng X, Lu H, et al. (2010) Angiogenesis of renal cell carcinoma: perfusion CT findings. Abdom Imaging 35: 622-628.

17. Squillaci E, Manenti G, Cicciò C, Nucera F, Bove P, et al. (2009) Perfusion-CT monitoring of cryo-ablated renal cells tumors. J exp clin cancer res 28: 138.

18. Kudo K, Sasaki M, Yamada K, Momoshima S, Utsunomiya H, et al. (2010) Differences in CT perfusion maps generated by different commercial software: quantitative analysis by using identical source data of acute stroke patients. Radiology 254: 200-209.

19. Sun H, Xue HD, Liu W, Wang Y, Zhao WM, et al. (2008) Perfusion Characteristics of Renal Mass with 64-slice Spiral Computed Tomography. CAMS 30: 680-685.

20. Ellika SK, Jain R, Patel SC, Scarpace L, Schultz LR, et al. (2007) Role of Perfusion CT in Glioma Grading and Comparison with Conventional MR Imaging Features. Am j neuroradiol 28: 1981-1987 